\title{
Neutrinos: In and Out of the Standard Model ${ }^{1}$
}

\author{
Stephen Parke \\ Theoretical Physics Department, \\ Fermi National Accelerator Laboratory, \\ P.O. Box 500, Batavia, IL 60510, USA \\ email:parke@fnal.gov
}

\begin{abstract}
The particle physics Standard Model has been tremendously successful in predicting the outcome of a large number of experiments. In this model Neutrinos are massless. Yet recent evidence points to the fact that neutrinos are massive particles with tiny masses compared to the other particles in the Standard Model. These tiny masses allow the neutrinos to change flavor and oscillate. In this series of Lectures, I will review the properties of Neutrinos In the Standard Model and then discuss the physics of Neutrinos Beyond the Standard Model. Topics to be covered include Neutrino Flavor Transformations and Oscillations, Majorana versus Dirac Neutrino Masses, the Seesaw Mechanism and Leptogenesis.
\end{abstract}

Keywords: Neutrinos

PACS: 14.60.Pq,25.30.Pt,28.41.-i

In the Standard Model the neutrinos, $\left(v_{e}, v_{\mu}, v_{\tau}\right)$, are massless and interact diagonally in flavor,

$$
\begin{array}{llll}
W^{+} & \rightarrow e^{+}+v_{e} & Z & \rightarrow v_{e}+\bar{v}_{e} \\
W^{+} \rightarrow \mu^{+}+v_{\mu} & Z & \rightarrow v_{\mu}+\bar{v}_{\mu} \\
W^{+} \rightarrow \tau^{+}+v_{\tau} & Z & \rightarrow v_{\tau}+\bar{v}_{\tau} .
\end{array}
$$

Since they travel at the speed of light, their character cannot change from production to detection. Therefore, in flavor terms, massless neutrinos are relatively uninteresting compared to quarks.

\section{NEUTRINO OSCILLATIONS IN VACUUM:}

If neutrinos have mass, then time passes for them and they can change character since they are not traveling at the speed of light. Typically, the neutrino states that interact with the $\mathrm{W}$ and $\mathrm{Z}$ bosons are not necessarily the states that propagate simply in time but they are related by a unitary matrix,

$$
\left(\begin{array}{l}
v_{\mu} \\
v_{\tau}
\end{array}\right)=\left(\begin{array}{cc}
\cos \theta & \sin \theta \\
-\sin \theta & \cos \theta
\end{array}\right)\left(\begin{array}{l}
v_{1} \\
v_{2}
\end{array}\right)
$$

\footnotetext{
${ }^{1}$ Lectures presented at the CINVESTAV Advanced Summer School on Physics 2006, July 10-15, 2006, Mexico City, Mexico
} 
where $\left(v_{\mu}, v_{\tau}\right)$ are the flavor states, eg $W^{+} \rightarrow \mu^{+}+v_{\mu}$ and $\left(v_{1}, v_{2}\right)$ are the mass eigenstates. The angle $\theta$ is the mixing angle to be determined experimentally and eventually explained by the theory of fermion masses. The mass eigenstates propagate in time as $\left|v_{j}\right\rangle \rightarrow e^{-i p_{j} \cdot x}\left|v_{j}\right\rangle \quad$ with $\quad p_{j}^{2}=m_{j}^{2}$. (The greek (latin) letters $\alpha, \beta \ldots(i, j \ldots)$ refer to flavor (mass) eigenstates.)

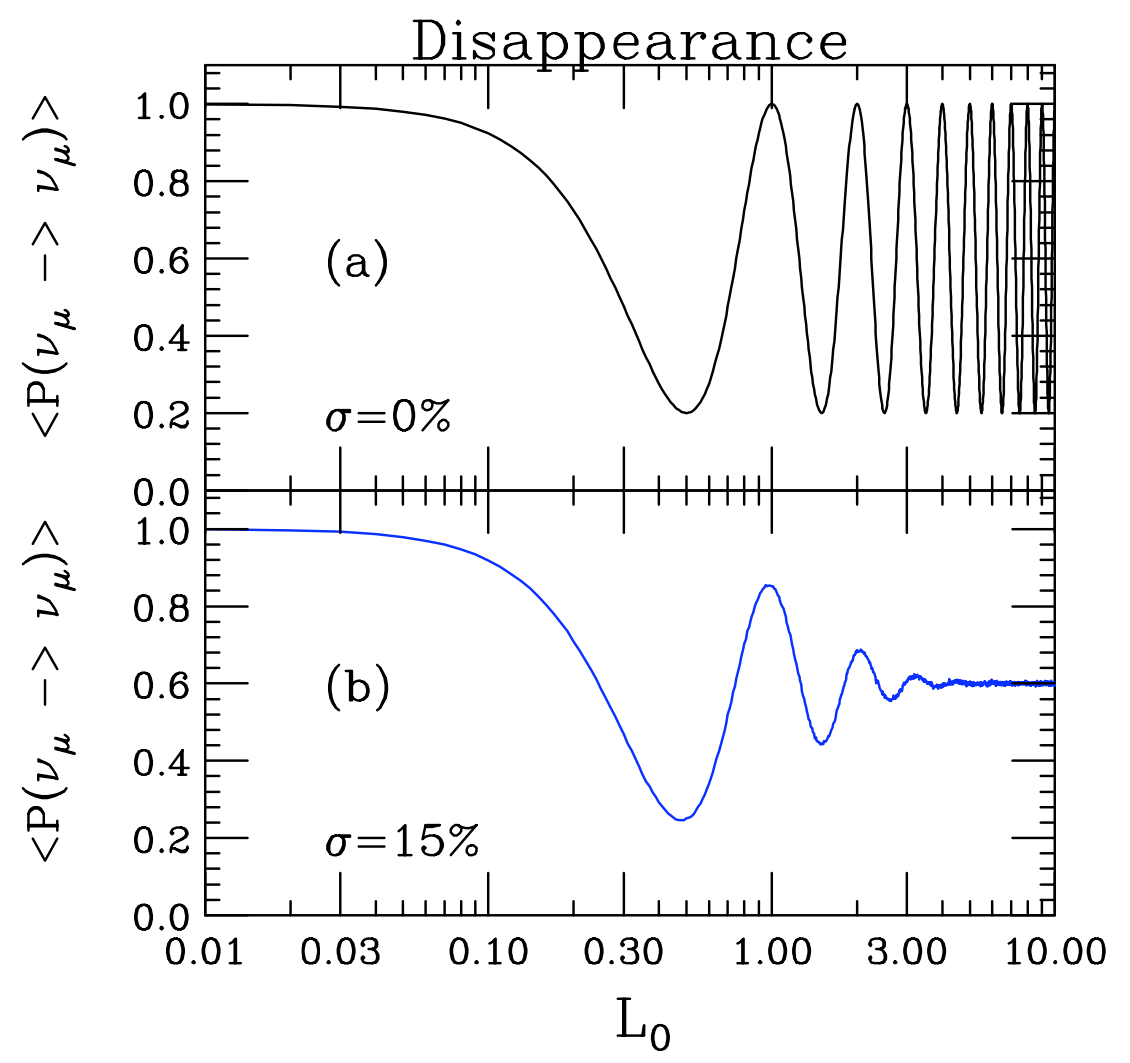

FIGURE 1. The survival probability for a muon neutrino versus distance traveled in units of the oscillation length, $4 \pi E / \delta \mathrm{m}^{2}$ : (a) for fixed neutrino energy, (b) using a gaussian energy spread equal to $15 \%$ of the mean energy of the neutrino. Notice that even for this narrow band beam the oscillations have disappeared after three oscillations!

Thus, the life of a neutrino can be represented as follows (at the amplitude level):

At Production: $\quad\left|v_{\mu}\right\rangle=\cos \theta\left|v_{1}\right\rangle+\sin \theta\left|v_{2}\right\rangle$

During Propagation: $\quad\left|v_{1}\right\rangle \rightarrow e^{-i p_{1} \cdot x}\left|v_{1}\right\rangle$ and $\left|v_{2}\right\rangle \rightarrow e^{-i p_{2} \cdot x}\left|v_{2}\right\rangle$

At Detection: $\left\{\begin{array}{l}\left|v_{1}\right\rangle=\cos \theta\left|v_{\mu}\right\rangle-\sin \theta\left|v_{\tau}\right\rangle \\ \left|v_{2}\right\rangle=\sin \theta\left|v_{\mu}\right\rangle+\cos \theta\left|v_{\tau}\right\rangle\end{array}\right.$

Thus, the transition probability for a neutrino to change flavor is

$$
P\left(v_{\mu} \rightarrow v_{\tau}\right)=\left|\cos \theta\left(e^{-i p_{1} \cdot x}\right)(-\sin \theta)+\sin \theta\left(e^{-i p_{2} \cdot x}\right) \cos \theta\right|^{2} .
$$


Using the same E formulation, we have that $p_{j}=\sqrt{E^{2}-m_{j}^{2}} \approx E-\frac{m_{j}^{2}}{2 E}$ and therefore

$$
P\left(v_{\mu} \rightarrow v_{\tau}\right)=\sin ^{2} \theta \cos ^{2} \theta\left|e^{-i m_{2}^{2} L / 2 E}-e^{-i m_{1}^{2} L / 2 E}\right|^{2}=\sin ^{2} 2 \theta \sin ^{2} \Delta
$$

where $\Delta \equiv \delta m^{2} L / 4 E$ is the kinematic phase, with $\delta m^{2}=m_{2}^{2}-m_{1}^{2}$. The disappearance probability is given by

$$
P\left(v_{\mu} \rightarrow v_{\mu}\right)=1-P\left(v_{\mu} \rightarrow v_{\tau}\right)=1-\sin ^{2} 2 \theta \sin ^{2} \Delta
$$

If we put the $\hbar$ 's and c's into the appearance probability we find

$$
P\left(v_{\mu} \rightarrow v_{\tau}\right)=\sin ^{2} 2 \theta \sin ^{2}\left(\frac{\delta m^{2} c^{4} L}{4 \hbar c E}\right)
$$

In the semi-classical limit, $\hbar \rightarrow 0$, the oscillation length goes to zero and the oscillations are averaged out. This is the same limit as letting $\delta m^{2}$ become large. This is precisely what happens in the quark sector. In Fig. 1 we have shown the oscillation probability for both fixed energy and a gaussian spread of $15 \%$ of the mean neutrino energy. Notice that oscillations are observable only for a limited range of distance. At small distance the simple flavor description is a good one. But at very large distance using the probability description with mass eigenstates works well since the oscillations are averaged out. The neutrino mass eigenstates are effectively incoherent. Thus, in terms of probabilities ${ }^{2}$

At Production: the fraction of $\left|v_{\mu}\right\rangle$ that is $\left|v_{1}\right\rangle$ is $\cos ^{2} \theta$ the fraction of $\left|v_{\mu}\right\rangle$ that is $\left|v_{2}\right\rangle$ is $\sin ^{2} \theta$

During Propagation: flavor fractions in $\left|v_{1}\right\rangle$ and $\left|v_{2}\right\rangle$ remain unchanged

At Detection: the fraction of $\left|v_{1}\right\rangle$ that is $\left|v_{\mu}\right\rangle$ is $\cos ^{2} \theta$ the fraction of $\left|v_{1}\right\rangle$ that is $\left|v_{\tau}\right\rangle$ is $\sin ^{2} \theta$ the fraction of $\left|v_{2}\right\rangle$ that is $\left|v_{\mu}\right\rangle$ is $\sin ^{2} \theta$ the fraction of $\left|v_{2}\right\rangle$ that is $\left|v_{\tau}\right\rangle$ is $\cos ^{2} \theta$

Thus, in the $v_{\mu}$ beam, the fraction of $v_{1}$ is $f_{1}=\cos ^{2} \theta$ and $v_{2}$ is $f_{2}=\sin ^{2} \theta$, independently of the neutrino energy, and the survival probability is

$$
\begin{aligned}
P\left(v_{\mu} \rightarrow v_{\mu}\right) & =f_{1} \cos ^{2} \theta+f_{2} \sin ^{2} \theta \\
& =\cos ^{4} \theta+\sin ^{4} \theta=1-\sin ^{2} 2 \theta\left\langle\sin ^{2} \Delta\right\rangle,
\end{aligned}
$$

since $\left\langle\sin ^{2} \Delta\right\rangle=1 / 2$. Notice that the full treatment given earlier is really only useful for distances around (1/5 to 5 times, say) the oscillation length, $L_{0}=4 \pi E / \delta m^{2}$. At small distance, the oscillations haven't built up enough to be significant, whereas as at the large distance the oscillations are average out.

\footnotetext{
${ }^{2} v_{\mu}$ is the neutrino produced in association with $\mu^{+}$.
} 

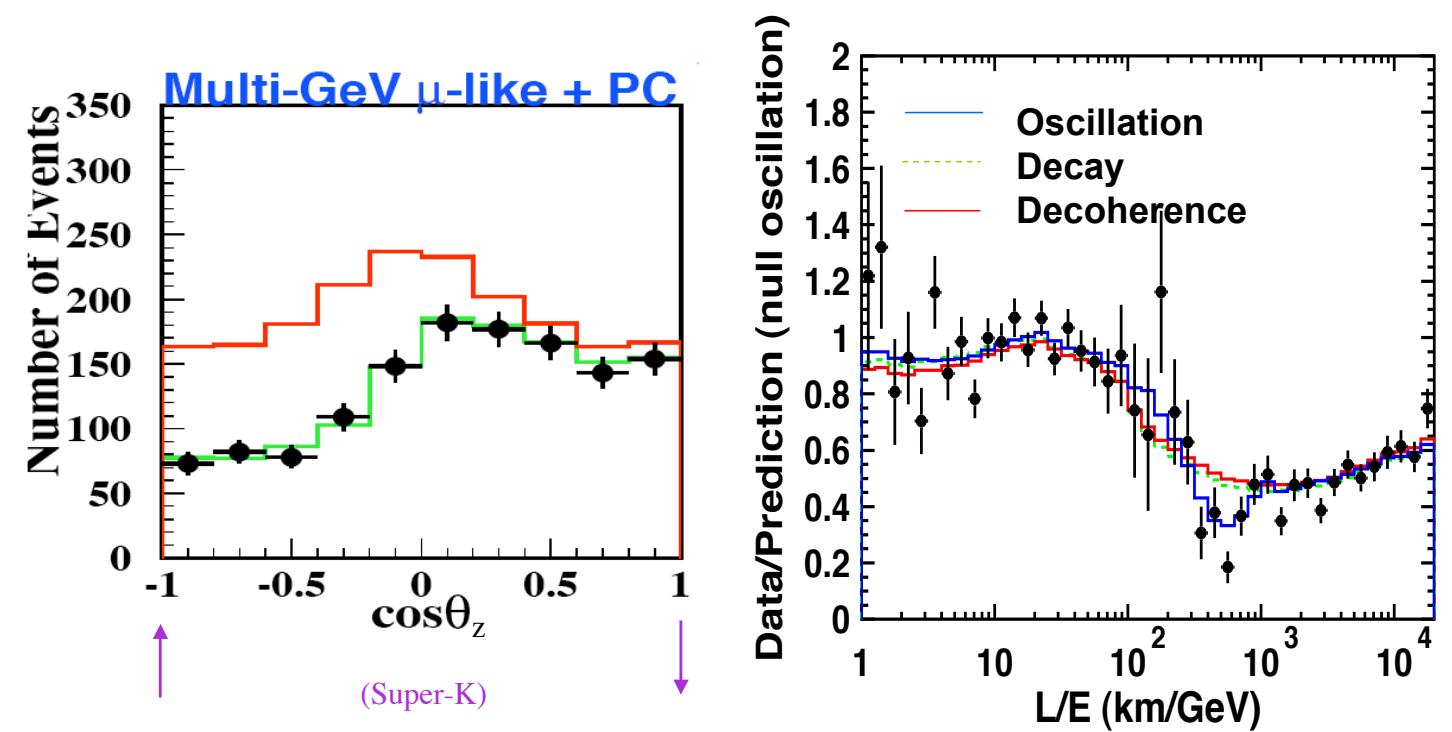

FIGURE 2. SuperKamiokande's evidence for neutrino oscillations both in the zenith angle and L/E plots.

\section{EVIDENCE FOR NEUTRINO OSCILLATIONS:}

\subsection{Atmospheric and Accelerator Neutrinos}

SuperKamiokande(SK) has very compelling evidence for $v_{\mu}$ disappearance in their atmospheric neutrino studies, see [1]. In Fig. 2 the zenith angle dependence of the multi$\mathrm{GeV} v_{\mu}$ sample is shown together with their L/E plot. This data fits very well the simple two component neutrino hypothesis with

$$
\delta m_{\text {atm }}^{2}=2-3 \times 10^{-3} \mathrm{eV}^{2} \quad \text { and } \quad \sin ^{2} \theta_{\text {atm }}=0.50 \pm 0.15
$$

This corresponds to a $\mathrm{L} / \mathrm{E}$ for oscillations of $500 \mathrm{~km} / \mathrm{GeV}$ and nearly maximal mixing. No evidence for the involvement of the $v_{e}$ is observed so the assumption is that $v_{\mu} \rightarrow v_{\tau}$.

Two beams of $v_{\mu}$ neutrinos have been sent to two detectors located at large distance: $\mathrm{K} 2 \mathrm{~K}$ experiment, [2], is from KEK to SK with a baseline of $250 \mathrm{~km}$ and the MINOS experiment, [3], from Fermilab to the Soudan mine with a baseline of $735 \mathrm{~km}$. Both experiments see evidence for $v_{\mu}$ disappearance which is summarized in Fig. 3

\subsection{Reactor and Solar Neutrinos:}

The KamLAND reactor experiment, [5], sees evidence for neutrino oscillations and not only at a different $\mathrm{L} / \mathrm{E}$ than the atmospheric and accelerator experiments but also this oscillation involves the $v_{e}$. These flavor transitions have also been seen in solar neutrino 


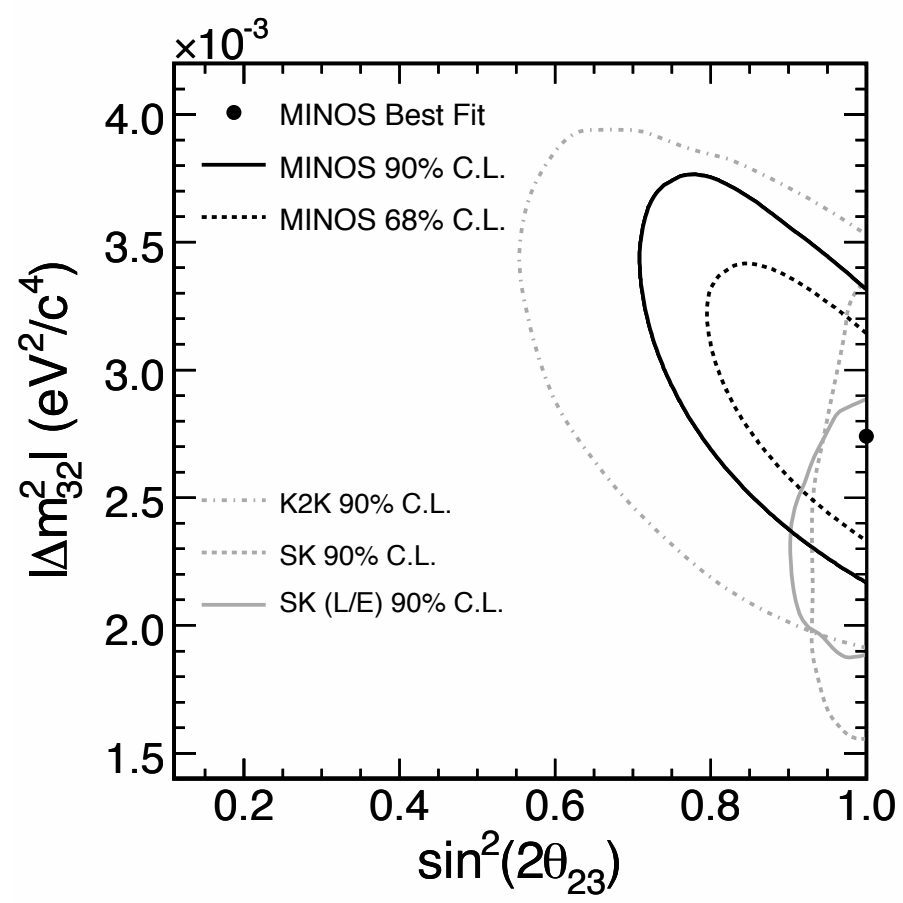

FIGURE 3. The allowed regions in the $\delta m_{a t m}^{2} \mathrm{v} \sin ^{2} \theta_{a t m}$ plane for MINOS data as well as for K2K data and two of the SK analyses. MINOS's best fit point is at $\sin ^{2} \theta_{a t m}=1$ and $\delta m_{a t m}^{2}=2.7 \times 10^{-3} \mathrm{eV}^{2}$.

experiments. The best fit values for $\delta m_{\odot}^{2}$ and $\sin ^{2} \theta_{\odot}$ are

$$
\delta m_{\odot}^{2}=8.0 \pm 0.4 \times 10^{-5} \mathrm{eV}^{2} \quad \text { and } \sin ^{2} \theta_{\odot}=0.31 \pm 0.03 .
$$

Thus, the $\mathrm{L} / \mathrm{E}$ for this oscillation is $15 \mathrm{~km} / \mathrm{MeV}$ which is 30 times larger than the atmospheric scale and the mixing angle, though large, is not maximal.

Fig. 4 shows the disappearance probability for the $\bar{v}_{e}$ from many reactor experiments as well as the flavor content of the ${ }^{8}$ Boron solar neutrino flux measured by SNO, [6], and SK, [7]. The reactor result can be understood in terms of vacuum neutrino oscillations and the fit to the disappearance probability, Eq. [4], suitably averaged over E and L, provides a good fit.

Solar neutrinos are somewhat more complicated because of the matter effects that the neutrinos experience from the production region until they exit the sun, at least for the ${ }^{8}$ Boron neutrinos. The pp and ${ }^{7} \mathrm{Be}$ neutrinos are little effected by the matter and undergo quasi-vacuum oscillations whereas the ${ }^{8}$ Boron neutrinos exit the sun mainly as a $v_{2}$ mass eigenstate because of matter effects and therefore do not undergo oscillations. This difference is primarily due to the difference in the energy of the neutrinos: $p p\left({ }^{7} \mathrm{Be}\right)$ have a mean energy of $0.2 \mathrm{MeV}(0.9 \mathrm{MeV})$ whereas ${ }^{8} \mathrm{~B}$ have a mean energy of $10 \mathrm{MeV}$ and the matter effect is proportional to energy of the neutrino.

The kinematic phase for solar neutrinos is

$$
\Delta_{\odot}=\frac{\delta m_{\odot}^{2} L}{4 E}=10^{7 \pm 1} .
$$



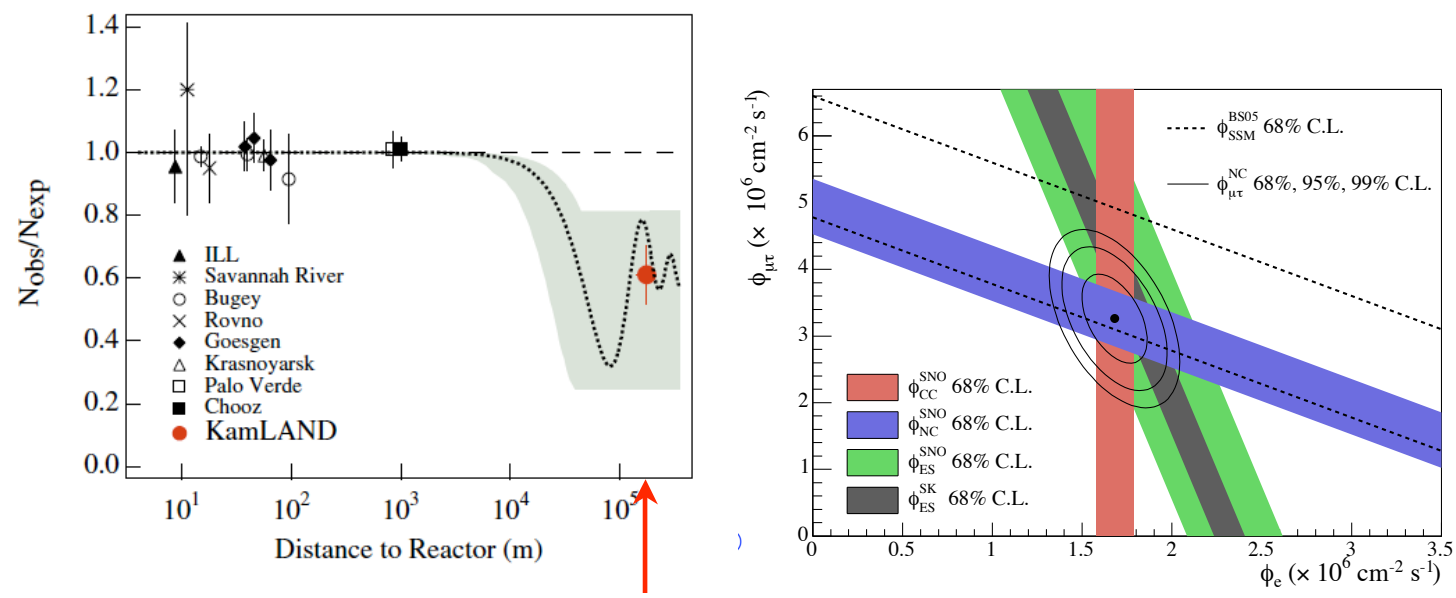

FIGURE 4. The disappearance of $\bar{v}_{e}$ observed by reactor experiments as a function of distance from the reactor. The flavor content of the ${ }^{8}$ Boron solar neutrinos for the various reactions for SNO and SK. CC: $v_{e}+d \rightarrow e^{-}+p+p, \mathrm{NC}: v_{x}+d \rightarrow v_{x}+p+n$ and $\mathrm{ES}: v_{\alpha}+e^{-} \rightarrow v_{\alpha}+e^{-}$.

Therefore, the solar neutrinos are "effectively incoherent" when they reach the earth. Hence the $v_{e}$ survival probability is given by $^{3}$

$$
\begin{aligned}
\left\langle P_{e e}\right\rangle & =f_{1} \cos ^{2} \theta_{\odot}+f_{2} \sin ^{2} \theta_{\odot} \\
\text { where } \quad f_{1}+f_{2} & =1 \text { and } \cos ^{2} \theta_{\odot}+\sin ^{2} \theta_{\odot}=1 .
\end{aligned}
$$

Now the pp and ${ }^{7} \mathrm{Be}$ solar neutrinos behave essentially as in vacuum and therefore $f_{1} \approx \cos ^{2} \theta_{\odot}=0.69$ and $f_{2} \approx \sin ^{2} \theta_{\odot}=0.31$ whereas the mass eigenstate fraction for the ${ }^{8} \mathrm{~B}$ are substantially different, see Fig. 5.

$$
\begin{aligned}
& \text { pp and }{ }^{7} \mathrm{Be} \\
& { }^{8} B
\end{aligned}
$$

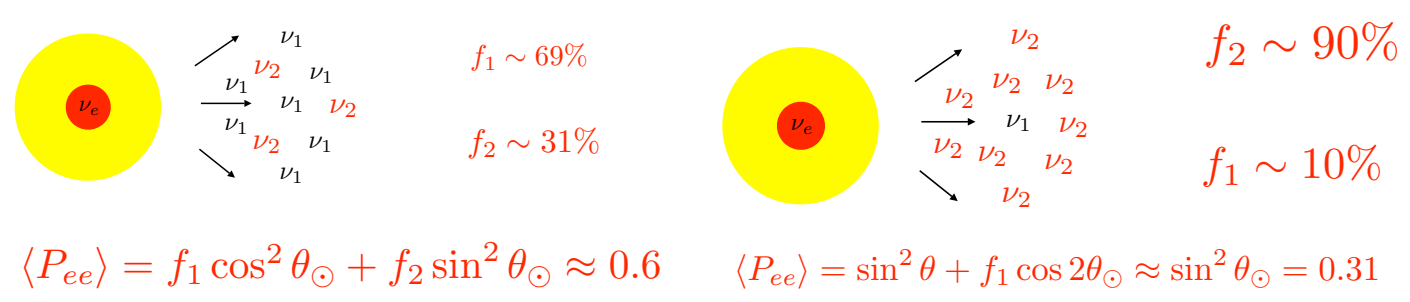

FIGURE 5. The sun produces $v_{e}$ in the core but once they exit the sun thinking about them in the mass eigenstate basis is useful. The fraction of $v_{1}$ and $v_{2}$ is energy dependent above $\sim 1 \mathrm{MeV}$ and has a dramatic effect on the ${ }^{8}$ Boron solar neutrinos, as first observed by Davis.

${ }^{3}$ Given the relationship between the quantities in this expression there are many equivalent ways to write the same expression. 

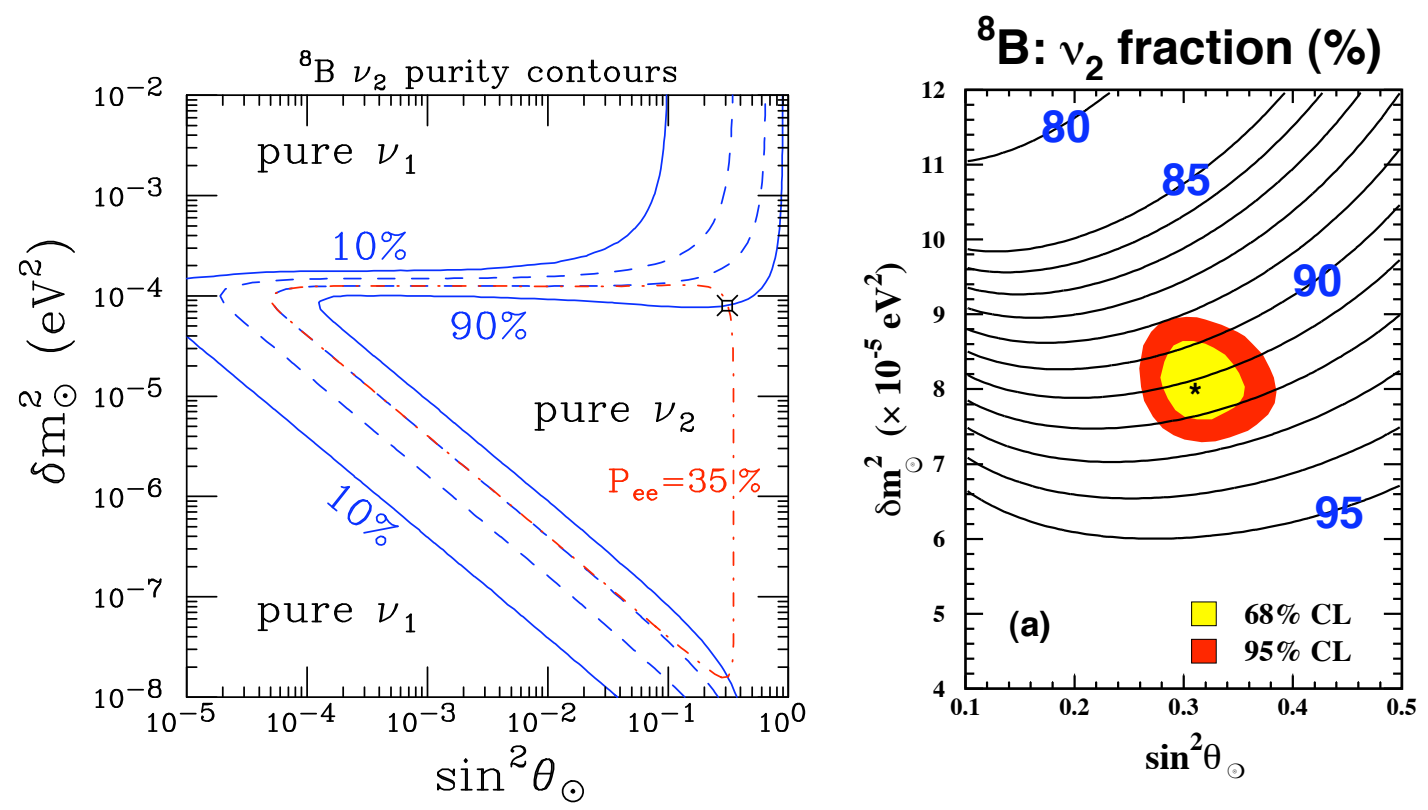

FIGURE 6. The $v_{2}$ fraction $(\%)$ in the $\delta m_{\odot}^{2}$ versus $\sin ^{2} \theta_{\odot}$ plane. (a) The solid and dashed (blue) lines are the $90,65,35$ and $10 \%$ iso-contours of the fraction of the solar ${ }^{8} \mathrm{~B}$ neutrinos that are $v_{2}$ 's. The current best fit value, indicated by the open circle with the cross, is close to the $90 \%$ contour. The iso-contour for an electron neutrino survival probability, $\left\langle P_{e e}\right\rangle$, equal to $35 \%$ is the dot-dashed (red) "triangle" formed by the $65 \% v_{2}$ purity contour for small $\sin ^{2} \theta_{\odot}$ and a vertical line in the pure $v_{2}$ region at $\sin ^{2} \theta_{\odot}=0.35$. Except at the top and bottom right hand corners of this triangle the $v_{2}$ purity is either $65 \%$ or $100 \%$. (b) Focuses in on the current allowed region. The 68 and 95\% CL are shown by the shaded areas with the best fit values indicated by the star using the combined fit of KamLAND and solar neutrino data given in [6] .

In a two neutrino analysis, the day-time $\mathrm{CC} / \mathrm{NC}$ of $\mathrm{SNO}$, which is equivalent to the day-time average $v_{e}$ survival probability, $\left\langle P_{e e}\right\rangle$, is given by

$$
\left.\frac{\mathrm{CC}}{\mathrm{NC}}\right|_{\text {day }}=\left\langle P_{e e}\right\rangle=f_{1} \cos ^{2} \theta_{\odot}+f_{2} \sin ^{2} \theta_{\odot},
$$

where $f_{1}$ and $f_{2}=1-f_{1}$ are understood to be the $v_{1}$ and $v_{2}$ fractions, respectively, averaged over the ${ }^{8} \mathrm{~B}$ neutrino energy spectrum weighted with the charged current cross section. Therefore, the $v_{1}$ fraction (or how much $f_{2}$ differs from $100 \%$ ) is given by

$$
f_{1}=\frac{\left(\left.\frac{\mathrm{CC}}{\mathrm{NC}}\right|_{\text {day }}-\sin ^{2} \theta_{\odot}\right)}{\cos 2 \theta_{\odot}}=\frac{(0.347-0.311)}{0.378} \approx 10 \pm ? ? \%,
$$

where the central values of the recent SNO analysis, [6], have been used. Due to the correlations in the uncertainties between the $\mathrm{CC} / \mathrm{NC}$ ratio and $\sin ^{2} \theta_{\odot}$ we are unable to estimate the uncertainty on $f_{1}$ from their analysis. Note, that if the fraction of $v_{2}$ were $100 \%$, then $\frac{\mathrm{CC}}{\mathrm{NC}}=\sin ^{2} \theta_{\odot}$.

Using the analytical analysis of the Mikheyev-Smirnov-Wolfenstein (MSW) effect given in Ref. [8], the mass eigenstate fractions are given by

$$
f_{2}=1-f_{1}=\left\langle\sin ^{2} \theta_{\odot}^{N}+P_{x} \cos 2 \theta_{\odot}^{N}\right\rangle_{8_{\mathrm{B}}},
$$




\section{Life of a Boron-8 Solar Neutrino:}

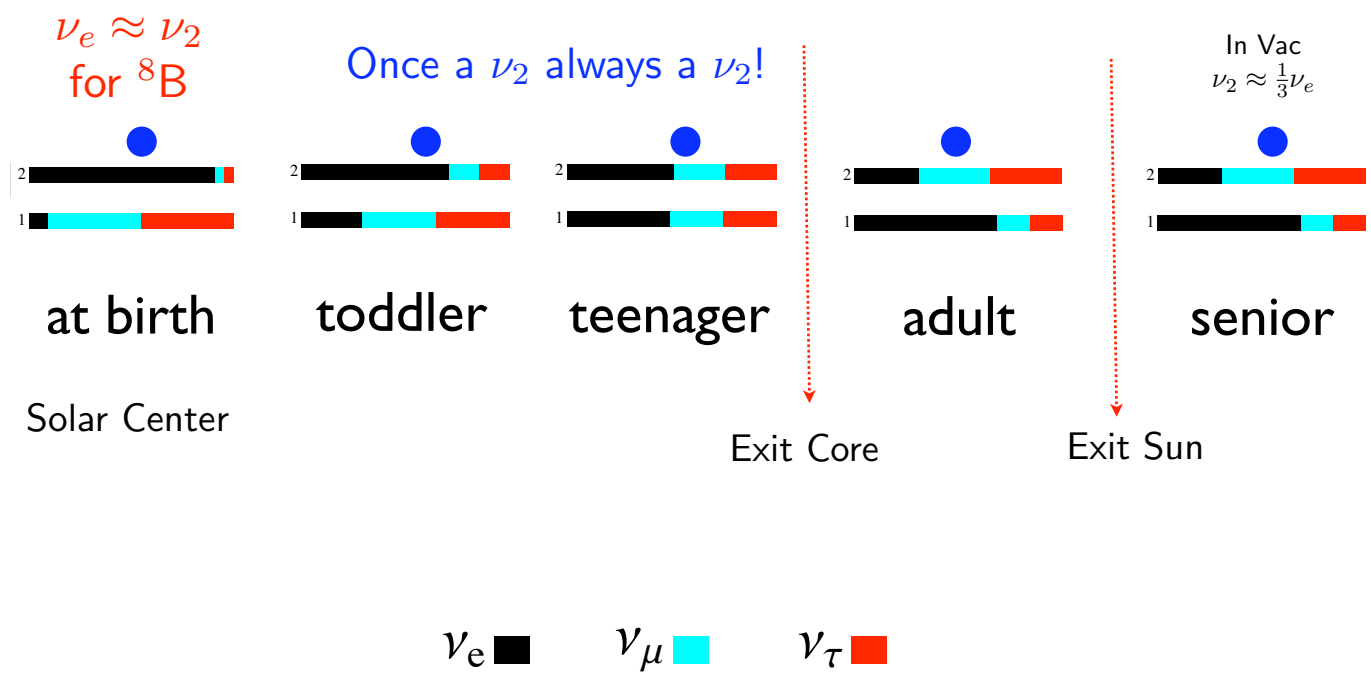

FIGURE 7. Life of a ${ }^{8}$ Boron solar neutrino from its birth at the center of the sun to its "death" in a detector at the earth. Notice how the flavor content of the the $v_{2}$ mass eigenstate evolves as the neutrino travels through the solar core.

where $\theta_{\odot}^{N}$ is the mixing angle defined at the $v_{e}$ production point and $P_{x}$ is the probability of the neutrino to jump from one mass eigenstate to the other during the MikheyevSmirnov resonance crossing. The average $\langle\cdots\rangle_{8_{B}}$ is over the electron density of the ${ }^{8} \mathrm{~B}$ $v_{e}$ production region in the center of the Sun predicted by the Standard Solar Model and the energy spectrum of ${ }^{8} \mathrm{~B}$ neutrinos weighted with SNO's charged current cross section. Fig. 6 shows the iso-contours of this averaged $v_{2}$ fraction using a threshold of $5.5 \mathrm{MeV}$ on the kinetic energy of the recoil electrons, this figure is taken from Ref. [9]. Thus, the ${ }^{8} \mathrm{~B}$ energy weighted average fraction of $v_{2}$ 's observed by $\mathrm{SNO}$ is

$$
f_{2}=91 \pm 2 \% \quad \text { at the } 95 \% \mathrm{CL} \text {. }
$$

Hence, the ${ }^{8} \mathrm{~B}$ solar neutrinos are the purest mass eigenstate neutrino beam known so far and SK famous picture of the sun taken with neutrinos is more than $80 \% v_{2}$ !!!

\section{NU STANDARD MODEL:}

The Neutrino Standard Model has emerged as follows ${ }^{4}$ :

- 3 light $\left(m_{i}<1 \mathrm{eV}\right)$ Majorana Neutrinos: $\Rightarrow$ only $2 \delta m^{2}$

${ }^{4}$ If MiniBooNE confirms the LSND result then this section will require major revision. 
$\left|\delta m_{\text {atm }}^{2}\right| \sim 2.5 \times 10^{-3} \mathrm{eV}^{2}$ and $\delta m_{\text {solar }}^{2} \sim+8.0 \times 10^{-5} \mathrm{eV}^{2}$

- Only three Active flavors (no steriles): $e, \mu, \tau$

- Unitary Mixing Matrix: 3 angles $\left(\theta_{12}, \theta_{23}, \theta_{13}\right), 1$ Dirac phase $(\delta), 2$ Majorana phases $(\alpha, \beta)$

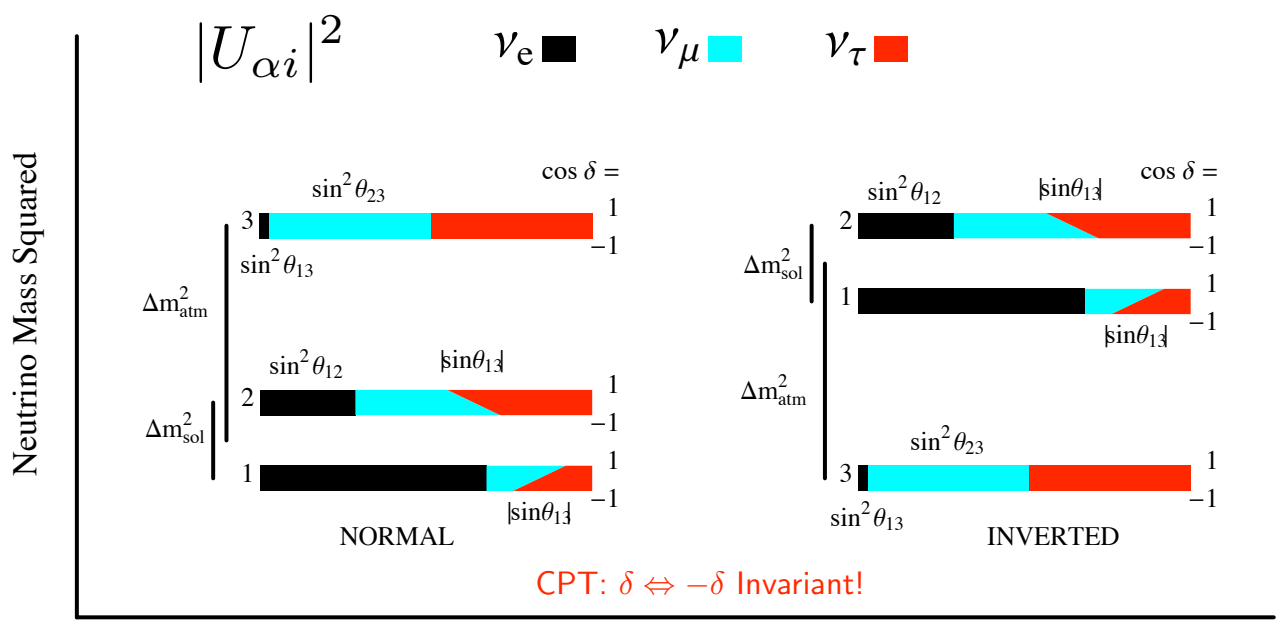

Fractional Flavor Content varying $\cos \delta$

FIGURE 8. Flavor content of the three neutrino mass eigenstates showing the dependence on the cosine of the CP violating phase, $\delta$. If CPT is conserved, the flavor content must be the same for neutrinos and anti-neutrinos. This figure was adapted from Ref. [10].

where the MNS mixing matrix relating flavor to mass eigenstates, $\left|v_{\alpha}\right\rangle=U_{\alpha i}\left|v_{i}\right\rangle$ is given by

$$
\begin{aligned}
& U_{\alpha i}=
\end{aligned}
$$

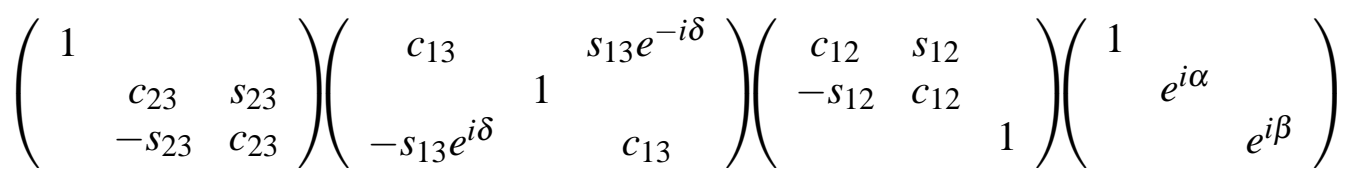

where $s_{i j}=\sin \theta_{i j}$ and $c_{i j}=\cos \theta_{i j}$. The (23) sector is identified with the atmospheric $\delta m_{\text {atm }}^{2}$ and the (12) sector is identified with the solar $\delta m_{\odot}^{2}$. The (13) sector is responsible for the $v_{e}$ flavor transitions at the atmospheric scale so far unobserved, see [11]. Therefore,

$$
\begin{aligned}
& \sin ^{2} \theta_{12}=0.31 \pm 0.03 \\
& \sin ^{2} \theta_{23}=0.50 \pm 0.15 \\
& \sin ^{2} \theta_{13}<0.04
\end{aligned}
$$


and the mass splittings ${ }^{5}$ are

$$
\left|\delta m_{32}^{2}\right|=2.7 \pm 0.4 \times 10^{-3} \mathrm{eV}^{2} \quad \text { and } \quad \delta m_{21}^{2}=+8.0 \pm 0.4 \times 10^{-5} \mathrm{eV}^{2}
$$

The mass of the lightest neutrino is unknown but the heaviest one must be lighter than about $1 \mathrm{eV}$. These mixing angles and mass splittings are summarized in Fig. 8 which also shows the dependence of the flavor fractions on the CP violating Dirac phase, $\delta$. The Majorana phases are unobservable in oscillations since oscillations depend on $U_{\alpha i}^{*} U_{\beta i}$ but they have observable CP conserving effects in neutrinoless double beta decay.

Asymmetry

Peaks:

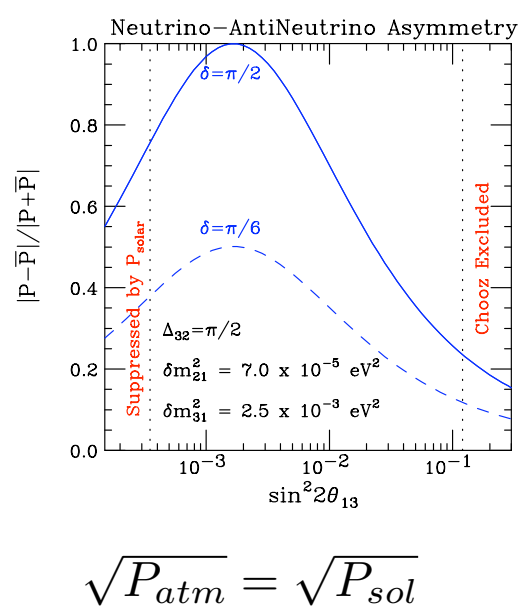

(a)
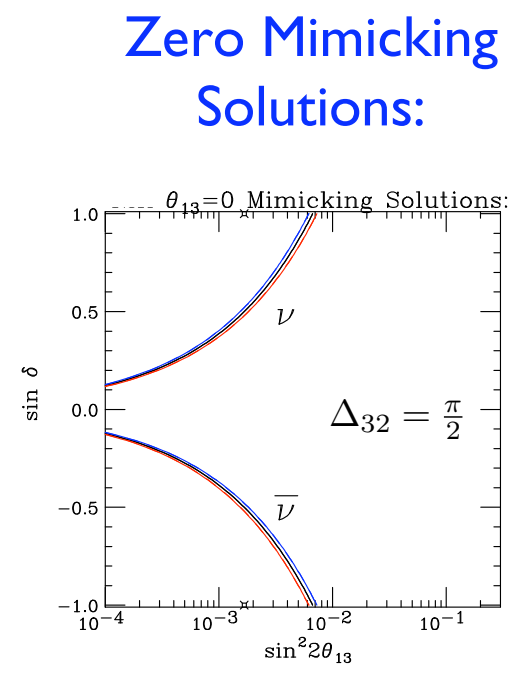

$$
\sqrt{P_{\text {atm }}}=-2 \sqrt{P_{\text {sol }}} \cos \left(\Delta_{32} \pm \delta\right)
$$

(b)

FIGURE 9. (a) The neutrino-antineutrino asymmetry as function of $\sin ^{2} 2 \theta_{13}$ at the first vacuum oscillation maximum. The asymmetry peaks when $\sin ^{2} 2 \theta_{13}=0.002$. (b) The zero mimicking solutions at the first vacuum oscillation maximum. Along these lines there is no evidence of non-zero $\theta_{13}$.

5 The $\delta m^{2}$ MINOS actually measures is

$$
\frac{\left(\left|U_{\mu 2}\right|^{2}\left|\delta m_{32}^{2}\right|+\left|U_{\mu 1}\right|^{2}\left|\delta m_{31}^{2}\right|\right)}{\left(\left|U_{\mu 2}\right|^{2}+\left|U_{\mu 1}\right|^{2}\right)} .
$$




\subsection{Genuine Three Flavor Effects: $v_{\mu} \rightarrow v_{\mathrm{e}}$}

The most likely genuine three flavor effects to be first observed are $v_{\mu} \rightarrow v_{e}$ and/or its $\mathrm{CP}$ and $\mathrm{T}$ conjugate processes. That is, in one of following transitions

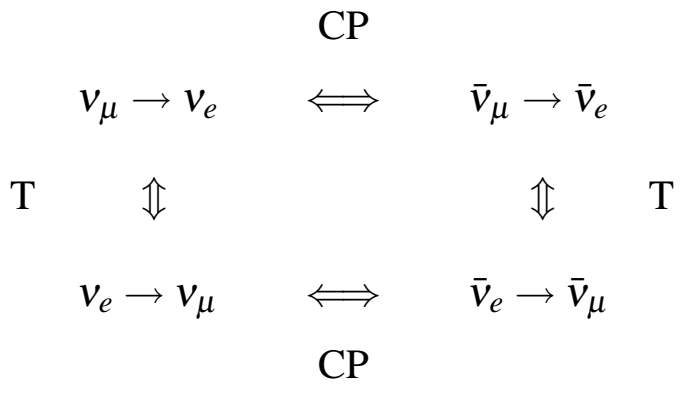

Processes across the diagonal are related by CPT. The first row will be explored in very powerful conventional beams, Superbeams, whereas the second row could be explored in Nu-Factories or Beta Beams.

In vacuum, the probability for $v_{\mu} \rightarrow v_{e}$ is derived like so, [12],

$$
\begin{aligned}
P\left(v_{\mu} \rightarrow v_{e}\right) & =\left|U_{\mu 1}^{*} e^{-i m_{1}^{2} L / 2 E} U_{e 1}+U_{\mu 2}^{*} e^{-i m_{2}^{2} L / 2 E} U_{e 2}+U_{\mu 3}^{*} e^{-i m_{3}^{2} L / 2 E} U_{e 3}\right|^{2} \\
& =\left|2 U_{\mu 3}^{*} U_{e 3} \sin \Delta_{31} e^{-i \Delta_{32}}+2 U_{\mu 2}^{*} U_{e 2} \sin \Delta_{21}\right|^{2} \\
& \approx\left|\sqrt{P_{a t m}} e^{-i\left(\Delta_{32}+\delta\right)}+\sqrt{P_{s o l}}\right|^{2}
\end{aligned}
$$

where $\sqrt{P_{a t m}}=\sin \theta_{23} \sin 2 \theta_{13} \sin \Delta_{31}$ and $\sqrt{P_{\text {sol }}} \approx \cos \theta_{23} \sin 2 \theta_{12} \sin \Delta_{21}$. For antineutrinos $\delta$ must be replaced with $-\delta$ and the interference term changes

$$
2 \sqrt{P_{a t m}} \sqrt{P_{\text {sol }}} \cos \left(\Delta_{32}+\delta\right) \Rightarrow 2 \sqrt{P_{a t m}} \sqrt{P_{\text {sol }}} \cos \left(\Delta_{32}-\delta\right)
$$

This allows for the possibility that $\mathrm{CP}$ violation maybe able to be observed in the neutrino sector since it allows for $P\left(v_{\mu} \rightarrow v_{e}\right) \neq P\left(\bar{v}_{\mu} \rightarrow \bar{v}_{e}\right)$.

In matter, $\sqrt{P_{\text {atm }}}$ and $\sqrt{P_{\text {sol }}}$ are modified as follows

$$
\begin{aligned}
& \sqrt{P_{\text {atm }}} \Rightarrow \sin \theta_{23} \sin 2 \theta_{13} \frac{\sin \left(\Delta_{31} \mp a L\right)}{\left(\Delta_{31} \mp a L\right)} \Delta_{31} \\
& \sqrt{P_{\text {sol }}} \Rightarrow \cos \theta_{23} \sin 2 \theta_{12} \frac{\sin (a L)}{(a L)} \Delta_{21}
\end{aligned}
$$

where $a= \pm G_{F} N_{e} / \sqrt{2} \approx(4000 \mathrm{~km})^{-1}$ and the sign is positive for neutrinos and negative for anti-neutrinos. This change follows since in both the (31) and (21) sectors the product $\left\{\delta m^{2} \sin 2 \theta\right\}$ is approximately independent of matter effects. In Fig. 10 the biprobability plots are shown for both T2K, [13], and NOvA, [14] . It is possible that these two experiments will determine the mass ordering (normal or inverted hierarchy, see Fig. reffig: pmns-sq), and observe $\mathrm{CP}$ violation in the neutrino sector. 

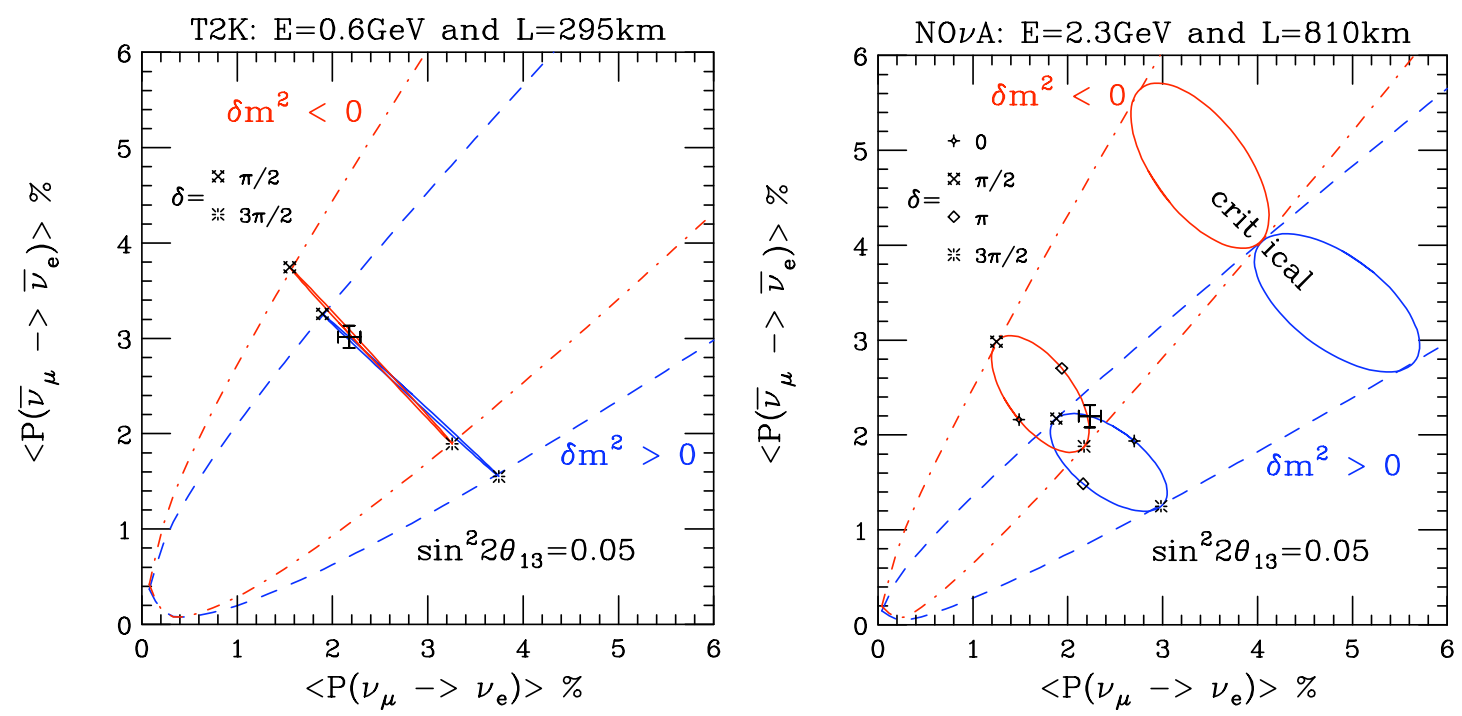

FIGURE 10. The bi-probability plots for both T2K and NOvA. The matter effects and hence the separation between the hierarchies is 3 times large for T2K than NOvA primarily due to the fact NOvA has three times the baseline as T2K. See [15] to understand how to use these plots to untangle CP violation and the mass hierarchy.

\section{NEUTRINO MASS}

\subsection{Absolute Neutrino Mass}

Tritium beta decay, neutrinoless double beta decay and cosmology all have the potential to provide us information on the absolute scale of neutrino mass. The Katrin tritium beta decay experiment, [16], has sensitivity down to $200 \mathrm{meV}$ for the "mass" of $v_{e}$ defined as

$$
m_{v_{e}}=\left|U_{e 1}\right|^{2} m_{1}+\left|U_{e 2}\right|^{2} m_{2}+\left|U_{e 3}\right|^{2} m_{3} .
$$

Neutrinoless double beta decay, see [17] for review, measures the following combination of neutrino mass,

$$
m_{\beta \beta}=\left|\sum m_{i} U_{e i}^{2}\right|=\left|m_{1} c_{13}^{2} c_{12}^{2}+m_{2} c_{13}^{2} s_{12}^{2} e^{2 i \alpha}+m_{3} s_{13}^{2} e^{2 i \beta}\right|,
$$

assuming the neutrinos are Majorana. It maybe possible to eventually reach below $10 \mathrm{meV}$ for $m_{\beta \beta}$ in double beta decay.

Cosmology measures the sum of the neutrino mases,

$$
m_{\text {cosmo }}=\sum_{i} m_{i} .
$$

If $\sum m_{i} \approx 50 \mathrm{eV}$ the universe's critical density would be saturated. The current limit, [18], is a few $\%$ of this number, $\sim 1 \mathrm{eV}$. Given the systematic uncertainties inherent in cosmology, a convincing limit of less than $100 \mathrm{meV}$ seems difficult. 
Fig. 11 shows the allowed values for these masses for both the normal and inverted hierarchy.
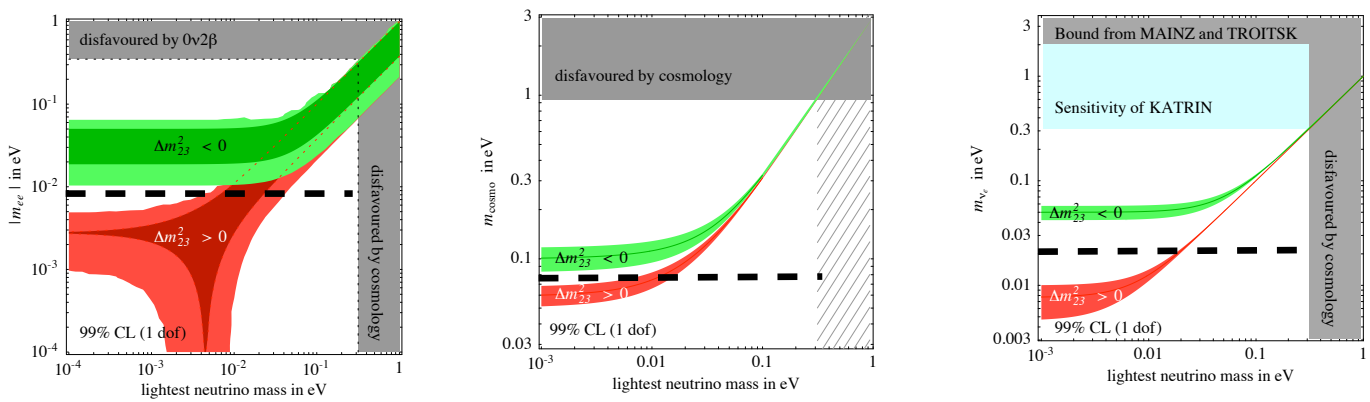

FIGURE 11. The "mass" measured in double $\beta$-decay, in cosmology and Tritium $\beta$-decay versus the mass of the lightest neutrino. Below the dashed lines, only the normal hierarchy is allowed. This figure was adapted from hep-ph/0503246 [19].

\subsection{Majorana v Dirac}

Fermion mass is a coupling of left handed to right handed states. Consider a massive fermion at rest, then one can consider this state as a linear combination of a massless right handed particle and a massless left handed particle as shown in Fig. 12. For a particle with an electric charge, like an electron, the left handed particle must have the same charge as the right handed particle. This is a Dirac mass. For a neutral particle, like a sterile neutrino, there is another possibility, the left handed particle could be coupled to the right handed anti-particle, this is the Majorana mass.

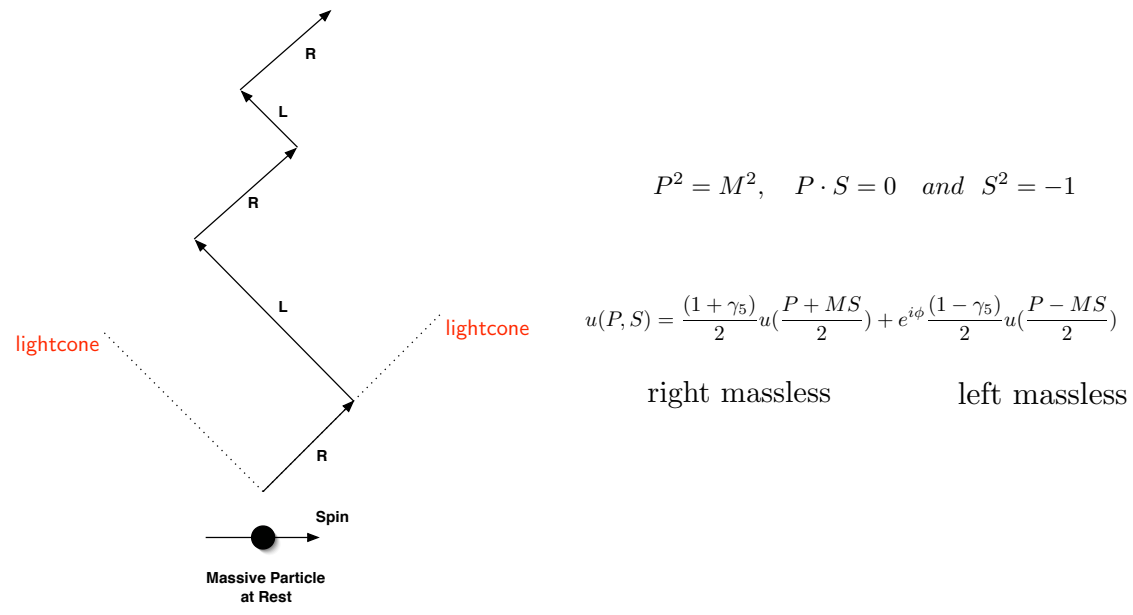

FIGURE 12. The left diagram shows how a massive particle at rest can be considered as a linear combination of two massless particles, one right handed and one left handed. The equation at the right shows the decomposition of a massive Dirac spinor into two massless spinors with different momenta, one right handed and the other left handed (from the Appendix of [20]). 
Therefore for a neutral particle there is the possibility of having both Dirac and Majorana masses, as

$\begin{array}{ccccc}\text { Left Chiral } & v_{L} & \Longleftrightarrow & \bar{v}_{R} & \\ & \mathbb{1} & & \mathbb{1} \text { Dirac Mass } \\ \text { Right Chiral } & v_{R} & \Longleftrightarrow & \bar{v}_{L} & \\ & & \text { Majorana } \\ & & \\ & & \text { Mass } & \end{array}$

For the neutrino, the left chiral field couples to $S U(2) \times U(1)$ therefore a Majorana mass term is forbidden by gauge symmetry. However, the right chiral field carries no quantum numbers. Therefore, the Majorana mass term is unprotected by any symmetry and it is expected to be very large. The Dirac mass terms are expected to be of the order of the charge lepton or quark masses. Thus, the mass matrix for the neutrinos is as in Fig. 13.
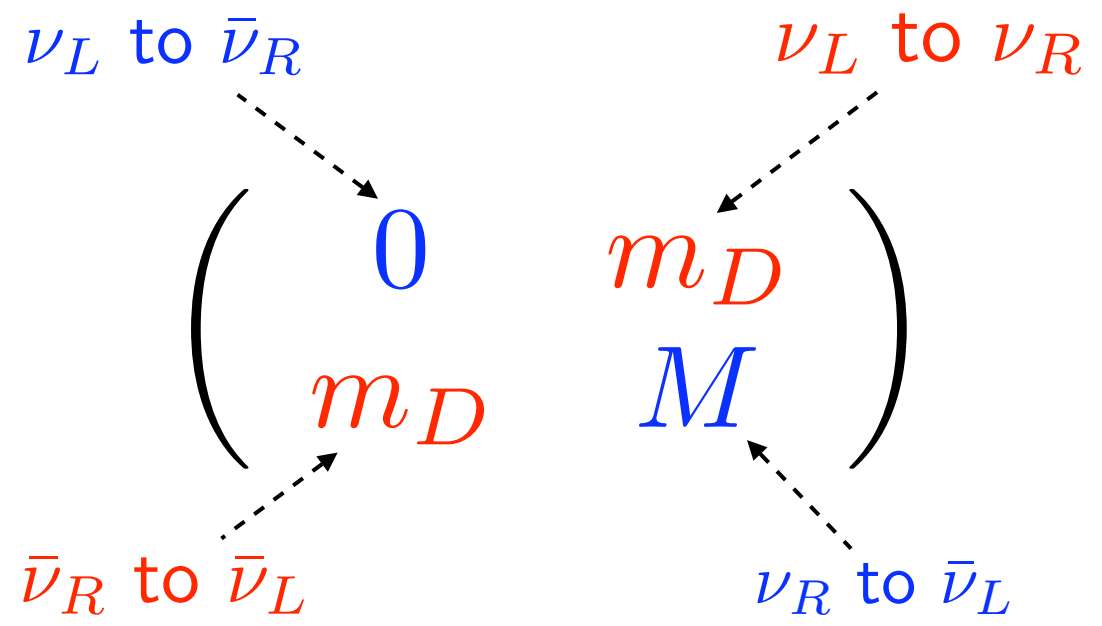

FIGURE 13. The neutrino mass matrix with the various right to left couplings. $m_{D}$ is the Dirac mass terms while 0 and $\mathrm{M}$ are Majorana masses for the charged and uncharged (under $S U(2) \times U(1)$ ) chiral components.

After diagonalizing the neutrino mass matrix, one is left with two Majorana neutrinos, one heavy Majorana neutrino with mass $\sim \mathrm{M}$ and one light Majorana neutrino with mass $m_{D}^{2} / M$. This is the famous seesaw mechanism, [21]. The light neutrino is the one observed in current experiments whereas the heavy neutrino is responsible for leptogenesis at very high energy scales since its decays are $\mathrm{CP}$ violating and depend on the Majorana phases in the MNS matrix, Eq. 16.

Majorana neutrinos not only allow for neutrinoless double beta decay but also for the possibility that the a muon neutrino, say, produces a positive charged muon, violating lepton number. However, this process would be suppressed by $\left(m_{v} / E\right)^{2}$ which is tiny, $10^{-20}$, and, therefore is unobservable. 


\title{
5. SUMMARY
}

\author{
Neutrino Mass $\Leftrightarrow$ Flavor Change
}

Open questions:

- Majorana v Dirac

- Light Steriles ???

- Mass Hierarchy $m_{3}>m_{2}>m_{1}$ OR $m_{2}>m_{1}>m_{3}$

(labeling such that $\left|U_{e 3}\right|^{2}<\left|U_{e 2}\right|^{2}<\left|U_{e 1}\right|^{2}$ )

- fraction of $v_{e}$ in $v_{3}(<4 \%)$ ( value of $\left.\sin ^{2} \theta_{13}\right)$

- Is CP violated ? $(\sin \delta \neq 0)$

- Mass of Heaviest Neutrino

- Mass of Lightest Neutrino

- New Interactions, Surprises !!!

\section{ACKNOWLEDGEMENTS}

I would like to thank all of the students and all of the organizers of the CINVESTAV Advanced Summer School for giving me the opportunity to present these lectures on neutrinos. I especially thank Chairman Omar Miranda for his wonderful hospitality. The support by Academia Mexicana de Ciencias (AMC-Mexico) and The United StatesMexico Foundation for Science (FUMEC) is acknowledged. Fermilab is operated by URA under DOE contract DE-AC02-76CH03000.

\section{REFERENCES}

1. Y. Ashie et al. [Super-Kamiokande Collaboration], Phys. Rev. D 71, 112005 (2005).

2. E. Aliu et al. [K2K Collaboration], Phys. Rev. Lett. 94, 081802 (2005);

M. H. Ahn et al. [K2K Collaboration], Phys. Rev. Lett. 90, 041801 (2003).

3. E. Ables et al. [MINOS Collaboration], FERMILAB-PROPOSAL-0875.

4. [MINOS Collaboration], arXiv:hep-ex/0607088.

5. K. Eguchi et al. [KamLAND Collaboration], Phys. Rev. Lett. 90, 021802 (2003). T. Araki et al. [KamLAND Collaboration], Phys. Rev. Lett. 94, 081801 (2005).

6. B. Aharmim et al. [SNO Collaboration], arXiv:nucl-ex/0502021.

7. S. Fukuda et al. [Super-Kamiokande Collaboration], Phys. Lett. B 539, 179 (2002).

8. S. J. Parke, Phys. Rev. Lett. 57, 1275 (1986); S. J. Parke and T. P. Walker, Phys. Rev. Lett. 57, 2322 (1986); S. J. Parke, "RESONANT NEUTRINO OSCILLATIONS," FERMILAB-CONF-86-131-T, Proceedings of theTopical Mtg. of 14th SLAC Summer Inst. on Particle Physics, Stanford, CA, Aug 5-8, 1986

9. H. Nunokawa, S. Parke and R. Z. Funchal, "What fraction of B-8 Solar neutrinos arrive at the Earth as a $v_{2}$ mass eigenstate", Fermilab-Pub-05-049, arXiv:hep-ph/0601198.

10. O. Mena and S. J. Parke, Phys. Rev. D 69, 117301 (2004) [arXiv:hep-ph/0312131].

11. M. Apollonio et al. [CHOOZ Collaboration], Phys. Lett. B 466, 415 (1999).

12. A. Cervera et al., Nucl. Phys. B 579, 17 (2000) [Erratum-ibid. B 593, 731 (2001)].

13. Y. Hayato et al., Letter of Intent, available at http://neutrino.kek.jp/jhfnu/

14. D. S. Ayres et al. [NOvA Collaboration], hep-ex/0503053. FERMILAB-PROPOSAL-0929, March 21, 2005. Revised NOvA Proposal available at http: //www-nova. fnal . gov/NOvA_ Proposal/Revised_NOvA_Proposal.html 
15. O. Mena and S. J. Parke, "Untangling CP violation and the mass hierarchy in long baseline experiments," Phys. Rev. D 70, 093011 (2004) [arXiv:hep-ph/0408070].

16. A. Osipowicz et al. [KATRIN Collaboration], "KATRIN: A next generation tritium beta decay experiment with sub-eV sensitivity for the electron neutrino mass," arXiv:hep-ex/0109033.

17. S. R. Elliott and P. Vogel, Ann. Rev. Nucl. Part. Sci. 52, 115 (2002) [arXiv:hep-ph/0202264].

18. D. N. Spergel et al., "Wilkinson Microwave Anisotropy Probe (WMAP) three year results: Implications for cosmology," arXiv:astro-ph/0603449.

19. A. Strumia and F. Vissani, Nucl. Phys. B 726, 294 (2005) [arXiv:hep-ph/0503246].

20. G. Mahlon and S. J. Parke, "Angular Correlations in Top Quark Pair Production and Decay at Hadron Phys. Rev. D 53, 4886 (1996) [arXiv:hep-ph/9512264].

21. M. Gell-Mann, P. Ramond and R. Slansky, in Supergravity, edited by P. van Nieuwenhuizen and D. Freedman, (North-Holland, 1979), p. 315;

T. Yanagida, in Proceedings of the Workshop on the Unified Theory and the Baryon Number in the Universe, edited by O. Sawada and A. Sugamoto (KEK Report No. 79-18, Tsukuba, 1979), p. 95;

R. N. Mohapatra and G. Senjanovic, Phys. Rev. Lett. 44, 912 (1980).

M. Fukugita and T. Yanagida, Phys. Lett. B 174, 45 (1986). 